Methods Carotid artery rings from 2-3 d-old and 9-10 d-old rats were mounted in myographs and studied at 33 and $37^{\circ} \mathrm{C}$.

Results Hypothermia did not significantly affect the contractions induced by $\mathrm{KCl}$ and $\mathrm{U} 46619$, nor the relaxations induced by acetylcholine (ACh), the nitric oxide (NO) donor sodium nitroprusside (SNP), the NO-independent stimulator of soluble guanylate cyclase (sGC) BAY 41-2272, the $\beta$-adrenoceptor agonist isoproterenol, the adenylate cyclase activator forskolin, and acute hypoxia $\left(\mathrm{PO}_{2} 3 \mathrm{kPa}\right)$. The relaxations induced by $\mathrm{ACh}$, isoproterenol, the $\beta_{2}$-adrenoceptor agonist salbutamol, the $\beta_{3}$-adrenoceptor agonist CL-316243 and hypoxia increased with postnatal age and were impaired by endothelium removal or by inhibition of NO synthase (L-NAME) or sGC (ODQ). By contrast, the relaxations induced by SNP, BAY 41-2272 and forskolin were endothelium-independent and did not change with age.

Conclusions Mild hypothermia $\left(33^{\circ} \mathrm{C}\right)$ does not affect the reactivity of neonatal rat carotid arteries. Our data suggest a reduced NO bioavailability in the carotid artery during the first days of life. This transient reduction in endothelium-dependent relaxation might play a role in the adaptation of the circulatory system to birth and in the neonatal vascular response to insults such as hypoxia.

\section{PS-341 LEARNING DIFFICULTIES AND UNDERNUTRITION. IS THERE A PROBLEM IN THE SYNAPSES? STUDYING IT WITH AN ANIMAL MODEL}

${ }^{1} \mathrm{C}$ Durán, ${ }^{2} \mathrm{C}$ Carrasco, ${ }^{2} \mathrm{~J}$ Rodriguez, ${ }^{3} \mathrm{~N}$ Villalmazo, ${ }^{3} \mathrm{JC}$ Jiménez-Chillarón, ${ }^{2} \mathrm{M}$ Camprubí. ${ }^{1}$ Neonatal Unit, Complejo Hospitalario Universitario de Vigo, Vigo, Spain; ${ }^{2}$ Neonatal Unit, Hospital Sant Joan de Deu, Barcelona, Spain; ${ }^{3}$ Fundación Sant Joan de Deu, Hospital Sant Joan de Deu, Barcelona, Spain

\subsection{6/archdischild-2014-307384.639}

Backgrounds and aims Intrauterine growth restriction (IUGR) and rapid postnatal weight gain increase susceptibility to metabolic syndrome during adult life. Longitudinal studies have also revealed high incidence of learning difficulties in children with IUGR.

The aim of the present study was to investigate the effect of nutrition on learning memory in an IUGR animal model.

Methods We use a mouse model of IUGR induced by caloric maternal undernutrition during late gestation. During the suckling period, dams were either fed ad libitum or food restricted. Pups were dived into: control-control (CC), undernutrition-control (UC), control-undernutriton (CU) and undernutrition-undernutrition (UU), indicating the prenatal-postnatal experimental conditions.

At 4 weeks of age, memory was assessed via water maze test. Finally, rats were anaesthetised and sacrified. To assess possible alterations of the hippocampal synaptic network, 3 specific synaptic proteins (PSD95, SNAP25, synaptophysin) were tested by Western Blot.

Results CC, UC, CU exhibited shorter escape latencies (EL) along the days. UU hardily changed its EL, indicating a poor spatial memory performance. Learning differences between CC and UU were statistical significant $(\mathrm{p}<0,01)$. CC animals had the higher protein synaptic levels in the hippocampus compared to all other groups $(\mathrm{p}<0,05)$.

Conclusions Nutrition plays an important role in learning. A poor pre and postnatal nutrition is associated with learning and memory alterations. Catch-up growth group showed an improvement in learning compared to UU. A decreased level of synaptic proteins in animals with a deficient nutrition (pre, postnatal or both), suggests that malnutrition results in less functional or efficient synapses.

\section{Preterm Brain Injury - Experimental}

\section{PS-341a NEW GENERATION LIPID EMULSION PROTECTS AGAINST LPS-INDUCED BRAIN INFLAMMATION IN PREMATURE PIGLETS}

${ }^{1} \mathrm{G}$ Guthrie, ${ }^{1} \mathrm{~B}$ Hodges, ${ }^{2} \mathrm{C}$ Martin, ${ }^{1} \mathrm{~B}$ Stoll, ${ }^{3} \mathrm{O}$ Olutoye, ${ }^{4} \mathrm{~S}$ Freedman, ${ }^{1} \mathrm{D}$ Burrin. ${ }^{\prime}$ Pediatrics, USDA/ARS Children's Nutrition Research Center/Baylor College of Medicine, Houston, USA; ${ }^{2}$ Neonatology, Beth Israel Deaconess Medical Center/Harvard Medical School, Boston, USA; ${ }^{3}$ Surgery, Texas Children's Hospital, Houston, USA; ${ }^{4}$ Medicine, Beth Israel Deaconess Medical Center/Harvard Medical School, Boston, USA

\subsection{6/archdischild-2014-307384.640}

Background Premature infants provided parenteral nutrition (PN) high in n-6 polyunsaturated fatty acids (PUFA) have increased risk of inflammatory disease, such as nosocomial sepsis. The pro-inflammatory insult can also contribute to injury and delayed neuronal growth in the perinatal brain. Provision of high long chain n-3 PUFA in parenteral lipids is associated with decreased inflammation and incidence of sepsis. The provision of n-3 PUFA, especially docosahexaenoic acid (DHA) also is critical for neurodevelopment in premature infants.

Aim To determine whether a new generation lipid emulsion high in n-3 PUFA (SMOFlipid) protects against inflammation and improves neuroprotection in response to lipopolysaccharide (LPS) compared to a lipid emulsion high in n-6 PUFA (Intralipid).

Methods Preterm piglets delivered $7 \mathrm{~d}$ preterm were assigned into two groups receiving complete TPN containing either Intralipid or SMOFlipid at $10 \mathrm{~g}^{*} \mathrm{~kg}^{-1 *} \mathrm{~d}^{-1}$ for $10 \mathrm{~d}$. On day 10 , subgroups of piglets were assigned to receive either an 8-hr infusion of lipopolysaccharide $(2 \mathrm{mg} / \mathrm{kg})$ or control saline and target gene expression in brain tissue was analysed.

Results LPS increased brain gene expression of pro-inflammatory cytokines IL-6, IL-8, and TNF in the Intralipid group, but not the SMOFlipid group. The gene expression of the antiinflammatory cytokine Il-10 was increased in both LPS-treated lipid groups. Brain-derived neuronal growth factor, a marker of neuronal proliferation, was deceased in the LPS-treated SMOFlipid group, but not the LPS-treated Intralipid group.

Conclusions SMOFlipid protected against LPS-induced inflammation, but did not acutely increase the expression of the neuroprotective protein, BDNF, in the presence of LPS.

\section{Primary Care General I}

\section{PS-342 MOTHER'S DEPRESSIVE SYMPTOMS AND THEIR COMMUNITIES' SOCIAL CAPITAL}

J Pascoe, R Rapp, M Perry, M Pesce. Pediatrics, Wright State University, Dayton, USA

\subsection{6/archdischild-2014-307384.641}

Background Previous studies have documented the association between mothers' personal social support and mothers' depressive symptoms. Maternal depressive symptoms have a pernicious effect on women's ability to function effectively as a mother. This study expands the concept of mothers' 'social connectedness' to include mothers' perception of their communities' support capital. 
Methods 612 mothers were recruited to participate in this cross sectional study from 11 community general paediatric practices. Social capital was assessed using the Social Capital Scale (SCS) and mothers' depressive symptoms were assessed using the Centre for Epidemiologic Depression Scale (CESD). The SCS has five factors. The local IRB approved this study.

Results About 3/4 of mothers were married or had a live in partner, $2 / 3$ were white, median family income was $\$ 30,000$, mothers' average years of school was 13 years. The total SCS score and scores for each of the five factors were all significantly inversely correlated (Spearman) with total score and the CESD ( $\mathrm{p}=$ 0.0001). Total CESD and total SCS $(r=-0.275)$. Belonging to the community factor was slightly stronger $(r=-302)$ than total SCS. While statistically significant, the remaining four factors all had correlations with the total CESD of around -0.2 or less.

Conclusions This study found that, beyond mothers' personal social network, a sense of belonging to the community is an important factor associated with mothers' depressive symptoms. Future studies should include longitudinal assessments of social capital and depressive symptoms to understand better the relation among personal social support, community social capital and depressive symptoms over time.

\section{PS-343 THE RELATIONSHIP BETWEEN PROLONGED QTC INTERVALS AND ANTIPSYCHOTIC DRUGS IN CHILDREN AND ADOLESCENTS: A RETROSPECTIVE CHART REVIEW}

${ }^{1} \mathrm{R}$ Kuge, ${ }^{2} \mathrm{~K}$ Miyazaki, ${ }^{3} \mathrm{M}$ Sakata, ${ }^{1} \mathrm{Y}$ Kikuchi, ${ }^{4} \mathrm{Y}$ Morikawa, ${ }^{2} \mathrm{~T}$ Ookura, ${ }^{2} \mathrm{~N}$ Kondo, ${ }^{5} \mathrm{M}$ Miura. 'Division of Psychology and Welfare, Tokyo Metropolitan Children's Medical Center, Tokyo, Japan; ${ }^{2}$ Division of Child and Adolescent Psychiatry, Tokyo Metropolitan Children's Medical Center, Tokyo, Japan; ${ }^{3}$ Division of Nursing, Tokyo Metropolitan Children's Medical Center, Tokyo, Japan; ${ }^{4}$ Division of Clinical Investigation, Tokyo Metropolitan Children's Medical Center, Tokyo, Japan; ${ }^{5}$ Division of Cardiology, Tokyo Metropolitan Children's Medical Center, Tokyo, Japan

10.1136/archdischild-2014-307384.642

Background Several psychotropic drugs are associated with the prolonged corrected QT interval (QTc) as measured on the electrocardiogram (ECG) that can lead to Torsades de Pointes (TdP) and sudden cardiac death. However, only few studies have evaluated the relationship between prolonged QTc intervals and administration of psychotropic drugs in children and adolescents. Therefore, the objectives of this study were to study the frequency of prolonged QTc intervals and to assess whether there exists a relationship between prolonged QTc intervals and the dose of antipsychotic drugs administered to adolescent psychiatric inpatients.

Methods The study subjects comprised 59 children and adolescents (15 male, 44 female) who were inpatients diagnosed as having F2 according to ICD-10 Classification of Mental and Behavioural Disorders at admission between April 2012 and March 2013. The average age of the subjects was $15.2 \pm 2.0$ years (range: 10.5-18.5 years). We retrospectively reviewed their medical records to characterise patient demographics, medication received, and QTc intervals on the ECG.

Results The average QTc interval recorded was $434.5 \pm 20.3$ msec (range: $391-487$ ). The QTc duration was $\geq 440 \mathrm{msec}$ (range: 442-487) for 21 patients (35.6\%). Chlorpromazine equivalents were similar between patients with normal and prolonged QTc values $(353.9 \pm 301.3$ vs. $420.6 \pm 348.8 ; \mathrm{p}=0.47)$. There were no patients with sudden death or syncope related to TdP.

Conclusions In this study, the frequency of prolonged QTc interval on admission was higher than that previously reported
$(1.97 \%)$, and it did not correlate with the current chlorpromazine equivalents. In the future, we intend to examine the frequency of prolonged QTc intervals among various psychiatric disorders.

\section{PS-344 EFFECTIVENESS OF PROGRAM "5 STEPS FOR HEALTH" IN SCHOLAR CHILDREN IN MEXICO"}

${ }^{1} \mathrm{M}$ Macias, ${ }^{1} \mathrm{C}$ Avila-Huerta, ${ }^{2} \mathrm{~J}$ De la Roca-Chiapas, ${ }^{1} \mathrm{M}$ Garay-Sevilla. ${ }^{1} \mathrm{C}$ iencias Medicas, Universidad de Guanajuato, Leon, Mexico; ${ }^{2}$ Psicologia, Universidad de Guanajuato, Leon, Mexico

\subsection{6/archdischild-2014-307384.643}

Background Obesity and sedentary lifestyle has increased in the last years. In Mexico, The Ministry of Education included information for children about physical activity and nutrition in elementary schools, in another hand, The Health Ministry suggest the program ' 5 steps for health' $(5 \mathrm{SH})$, which is based in theory to change habits.

Aim Investigate the change to healthy habits in children 10 to 12 years old comparing traditional education Vs ' $5 \mathrm{SH}$ '

Methods Children were randomly assigned to traditional education group ( $\mathrm{n}=101,1$ session/week) or $5 \mathrm{SH}$ group $(\mathrm{n}=134,2$ sessions/week) and followed for 6 months; and six months later were interviewed to verify the change of habits. At the beginning and the end of study physical activity was recorder by survey, weight and height were measured and classified as normal, overweight or obese according Body Mass Index (BMI).

Results At beginning of study both groups were similar, and one year of follow-up we found more overweight and obese in the children with traditional education vs $5 \mathrm{SH} 34.7 \%$ and $5.9 \%$ vs $17.2 \%$ and $3.0 \%$, respectively $\left(X^{2}=11.2 ; \mathrm{p}=0.003\right)$. Physical activity increased in $5 \mathrm{SH}$ group from $124 \pm 124 \mathrm{~min} /$ week to $232 \pm 113 \mathrm{~min} / \mathrm{week} ;(\mathrm{p}<0.001)$ than traditional group from $131 \pm 128 \mathrm{~min} /$ week to $177 \pm 130 \mathrm{~min} /$ week; $(\mathrm{p}>0.05)$.

Conclusions The program ' 5 steps for health' focused in change of habits is more effective than traditional education to increase physical activity and prevent obesity.

\section{PS-345 REDUCTION OF PAEDIATRIC EMERGENCY HOSPITAL ADMISSIONS BY MODIFICATIONS IN PAEDIATRIC EMERGENCY DEPARTMENT POLICY}

A Elhassanien. Pediatrics, Mansoura Faculty of Medicine, Mansoura, Egypt

\subsection{6/archdischild-2014-307384.644}

Background Reduction in admissions is an important aim of emergency department working policy to overcome the problems of a shortage of inpatient beds, rising costs and exhausted resources. A new policy was instituted in the paediatric emergency department (PED) of a hospital in Kuwait with the following components: (1) assigning senior doctor staff (2) implementation of new disease management guidelines; and (3) maximising the useof the paediatric emergency department observation unit.

Objective To evaluate the effect of change in our policy on the admission rate.

Methods The effects of this policy on reduction of admission rates for total paediatric admissions and for some selected common paediatric conditions were prospectively studied over a period of 3 years from institution of the policy and compared with the 3-year period before the policy was instituted. 